\title{
Higgs production in the VH mode at ATLAS and CMS
}

\author{
Luca Mastrolorenzo*† \\ Rheinisch-Westfälische Technische Hochschule Aachen, Germany \\ E-mail: luca.mastrolorenzodcern.ch
}

\begin{abstract}
In these proceedings, the key role played by the Higgs boson associated production (VH) mode in the characterization of the electroweak spontaneous symmetry breaking mechanism is described through a review of the latest results from the ATLAS and CMS experiments obtained with the data collected during LHC Run 2. A focus is given to the recent discovery of the Higgs boson decay to a bottom quark-antiquark pair by ATLAS and CMS achieved through the analysis of the additional data collected during 2017. A review of the ATLAS and CMS searches for $\mathrm{VH}(\mathrm{H} \rightarrow \mathrm{WW})$ is also provided together with a summary of the role played by the $\mathrm{VH}$ production mechanism in the $\mathrm{H} \rightarrow \tau \tau$ observation carried out by CMS and in the challenging search for the Higgs boson decay to charm quarks published by ATLAS in the beginning of LHC Run 2.
\end{abstract}

7th Annual Conference on Large Hadron Collider Physics - LHCP2019

20-25 May, 2019

Puebla, Mexico

* Speaker.

${ }^{\dagger}$ on behalf of the ATLAS and CMS Collaborations 


\section{Introduction}

The characterization of the nature of the Higgs boson $(\mathrm{H})$ and its properties currently constitutes one of the top priorities of the particle physics community. In particular, the Higgs boson decays and production modes can provide further insights into the understanding of the electroweak spontaneous symmetry breaking mechanism [1, 2, 3], through which elementary particles acquire their mass. Back in 2012, the Higgs boson discovery [4, 5, 6] has been achieved observing the Higgs boson decays into gauge bosons (ZZ, $\gamma \gamma$ and WW, even if this latter has not achieved an observed significance above five standard deviations with the LHC Run-1 data). It was only three years later that the ATLAS and CMS searches, combined, led to the observation of the first fermionic Higgs boson decay: $\mathrm{H} \rightarrow \tau \tau$. To fully characterize the Higgs sector, all the couplings of the Higgs boson with the Standard Model (SM) particles must be measured, including the selfinteractions of the Higgs field. This challenge begun during Run-1 with analyses carried out by the ATLAS and CMS collaborations aimed at observing the Yukawa couplings of the Higgs boson with third-generation quarks, discovering the $\mathrm{ttH}[7,8]$ and $\mathrm{H} \rightarrow \mathrm{b} \overline{\mathrm{b}}[9,10]$ processes with the additional data collected during LHC Run 2 (until 2017). However, searches for the aforementioned processes have required a considerable development of the analysis strategies, massively introducing the exploitation of multivariate analysis techniques such as deep neural networks, used in different stages of data analysis. Another important objective to be pursued during LHC Run 2 consists in improving the characterization of the Higgs boson production processes. It is for this reason that the production of a Higgs boson with a vector boson (associated production, $\mathrm{VH}$ ) plays a pivotal role both in providing a more precise measurement of the Higgs boson production mechanism (as demonstrated by the observation of $\mathrm{H} \rightarrow \mathrm{b} \overline{\mathrm{b}}$ in 2018) and in the exploration of the various decays of the Higgs boson that remain to be assessed. In particular, searches for the $\mathrm{H} \rightarrow \mathrm{c} \bar{c}$ decay mode would greatly benefit from the exploitation of the VH production mechanism. Indeed, the leptonic decays of the vector boson provide a crucial handle to collect the events efficiently at the trigger level and to reduce considerably the overwhelming QCD background. Moreover, a requirement on the vector boson transverse momentum further reduces the background contamination from $\mathrm{V}+\mathrm{jets}$ processes, characterized by a rapidly decreasing vector boson $\mathrm{p}_{\mathrm{T}}$ spectrum. Also, the angular correlation between the vector boson and the Higgs boson directions can be exploited to further identify the signal events. These proceedings provide a summary of the main measurements carried out by the ATLAS and CMS collaborations targeting the associated production of the Higgs boson with a vector boson carried out during LHC Run 2. A detailed description of the CMS and ATLAS detectors, together with a definition of the coordinate system and the relevant kinematic variables, can be found in $[11,12]$.

\section{Higgs boson decay to bottom quarks}

In 2018 both the ATLAS and CMS collaborations observed for the first time the decay of a Higgs boson into a bottom quark-antiquark pair $[9,10]$. The discovery of this Higgs boson decay mode is of utmost importance in the characterization of the spontaneous symmetry breaking mechanism. Indeed, this is the Higgs boson decay mode with the highest branching fraction predicted by the SM [13] and the precision of its measurement limits the sensitivity to possible contribution 
from physics beyond the SM (BSM). The search for the $\mathrm{H} \rightarrow \mathrm{b} \overline{\mathrm{b}}$ decay mode can benefit from a large signal events yield, given the highest branching fraction among all the other decays of the Higgs boson. Nevertheless, analyses targeting the $\mathrm{H} \rightarrow \mathrm{b} \overline{\mathrm{b}}$ decay are characterized by a modest signal over background ratio. The experimental signature of the $\mathrm{H} \rightarrow \mathrm{b} \overline{\mathrm{b}}$ decay consists of two jets originating from the hadronization of the two bottom quarks coming from the Higgs boson. This makes the search for the $\mathrm{H} \rightarrow \mathrm{b} \bar{b}$ decay very challenging to be pursued at a hadron collider, due to the overwhelming background originating from QCD multijet events. From the ATLAS and CMS results, that led to the observation of the $\mathrm{H} \rightarrow \mathrm{b} \bar{b}$ decay mode by the two experiments independently, it has been found that the product of the VH cross-section and $\mathrm{H} \rightarrow \mathrm{b} \overline{\mathrm{b}}$ branching ratio is fully compatible with the SM prediction, as shown in Fig. 1. The total sensitivity to the $\mathrm{H} \rightarrow \mathrm{b} \overline{\mathrm{b}}$ process is driven mainly by the analysis of those events with a Higgs boson candidate produced in association with a vector boson as shown in Fig. 2. Indeed, the exploitation of the VH production mode is crucial to reduce the background contamination, as explained in Sec. 1. The ATLAS and CMS analysis strategies are summarised in Sec. 2.1 and 2.2. The dominant background processes after the event selection are $\mathrm{V}+\mathrm{jets}$, $\mathrm{t}+\mathrm{jets}$, single-top and diboson process.
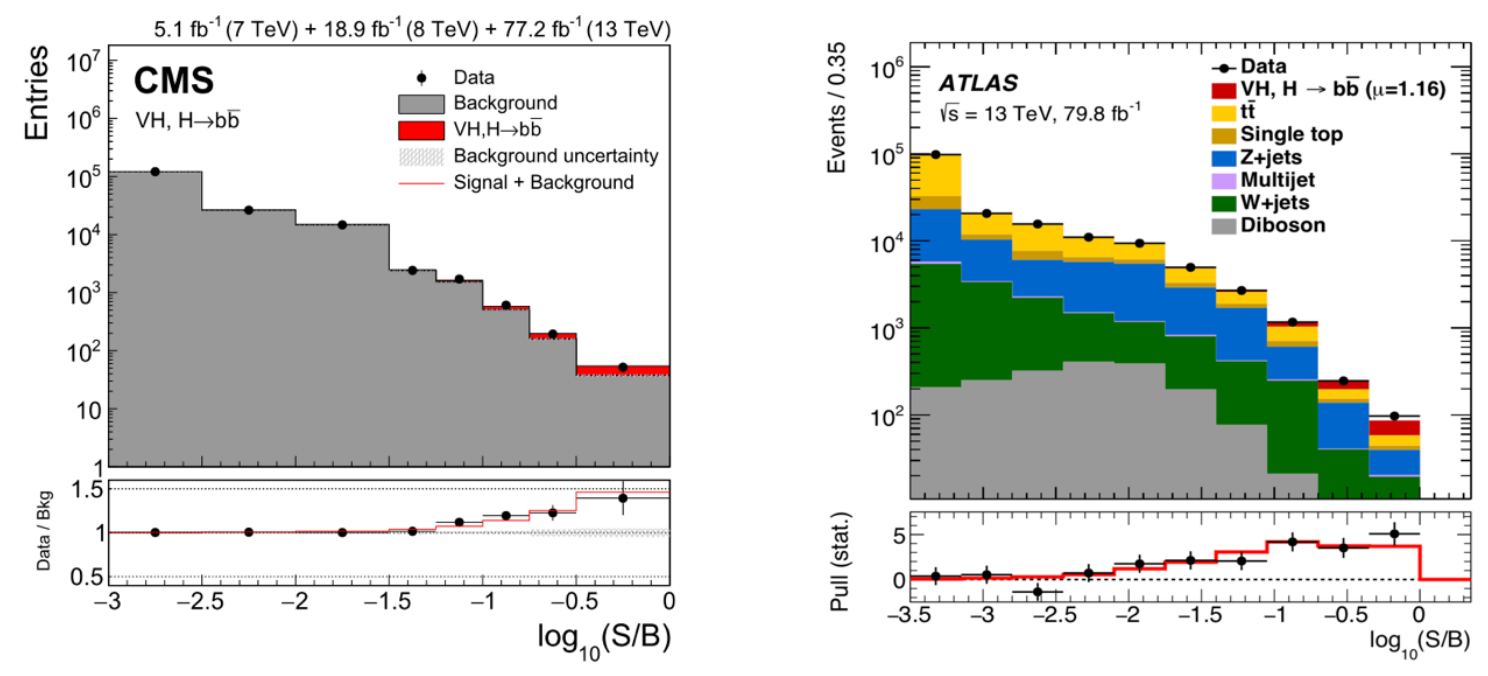

Figure 1: Left: distributions of signal, background, and data event yields sorted into bins of $\log _{10}(\mathrm{~S} / \mathrm{B})$, as given by the result of the fit to their corresponding multivariate discriminant in the CMS analysis. All events in the $\mathrm{VH}(\mathrm{H} \rightarrow \mathrm{b} \bar{b})$ signal regions of the combined Run-1 and Run 2 data sets are included. The red histogram indicates the Higgs boson signal contribution, while the grey histogram is the sum of all background yields. The bottom panel shows the ratio of the data to the background, with the total uncertainty in the background yield indicated by the grey hatching. The red line indicates the sum of signal plus background contribution divided by the background yield [10]. Right: Event yields as a function of $\log _{10}(\mathrm{~S} / \mathrm{B})$ for data, background and a Higgs boson signal with $\mathrm{m}_{\mathrm{H}}=125 \mathrm{GeV}$ selected by the ATLAS analysis. Finaldiscriminant bins in all regions are combined into bins of $\log _{10}(\mathrm{~S} / \mathrm{B})$, with $\mathrm{S}$ being the fitted signal and $\mathrm{B}$ the fitted background yields. In the lower panel, the pull of the data relative to the background is shown with statistical uncertainties only [9].

\subsection{CMS search for $\mathrm{VH}(\mathrm{H} \rightarrow \mathrm{b} \overline{\mathrm{b}})$}

The CMS search for $\mathrm{VH}(\mathrm{H} \rightarrow \mathrm{b} \bar{b})$ carried out by the CMS collaboration relies on a categorization of the selected events according to the number of reconstructed electrons (e) and muons 

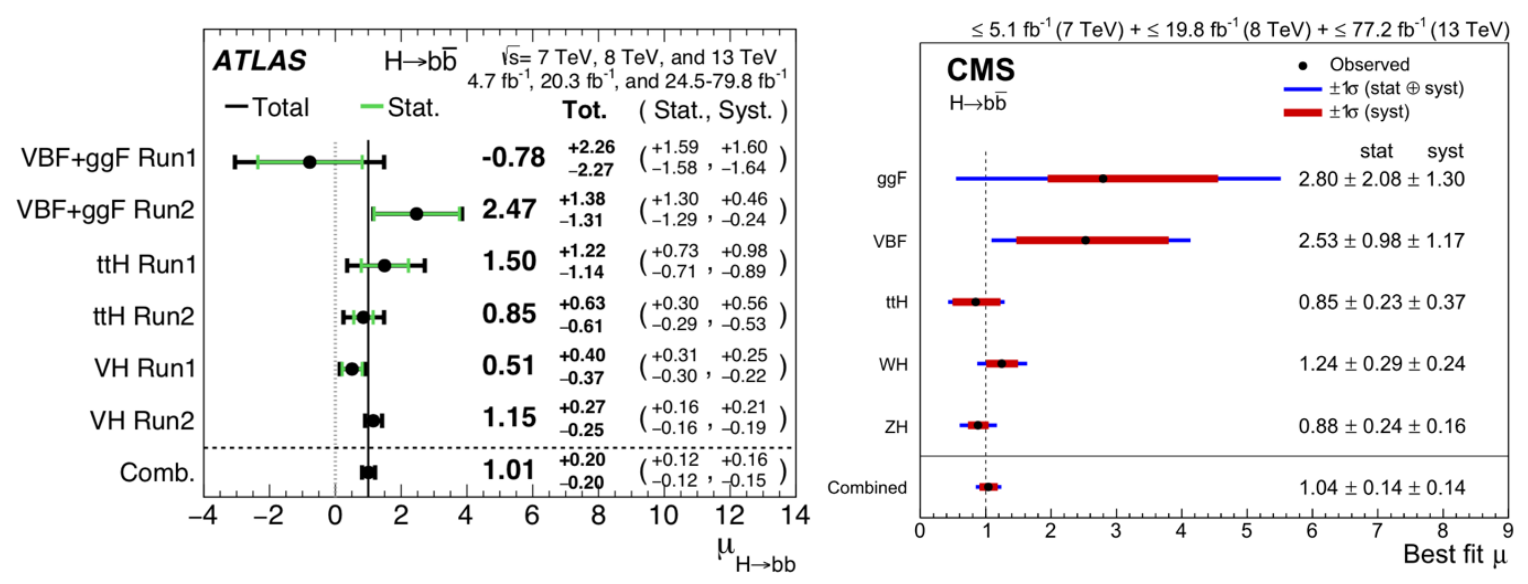

Figure 2: ATLAS [9] and CMS [10] results for the best fit value of the $\mathrm{H} \rightarrow \mathrm{b} \overline{\mathrm{b}}$ signal strength with its $1 \sigma$ systematic (red for CMS and green for ATLAS) and total (blue for CMS and black for ATLAS) uncertainties for the five individual production modes considered, as well as the overall combined result. The vertical dashed line (CMS) and continuous line (ATLAS) indicates the SM expectation. All results are extracted from a single fit combining all input analyses, with $\mathrm{m}_{\mathrm{H}}=125 \mathrm{GeV}$.

$(\mu)$ passing the trigger selection, offline $\mathrm{p}_{\mathrm{T}}$ and $\eta$ requirements and satisfying quality criteria, as well as on the presence of missing transverse energy. Also the presence of at least 2 reconstructed jets with a $\mathrm{p}_{\mathrm{T}}>20 \mathrm{GeV}$ is required. In CMS the physics object reconstruction is achieved through the particle-flow algorithm [14], which aims to reconstruct and identify each particle in an event, with an optimized combination of information from the various elements of the CMS detector. The energy of photons is obtained from the ECAL measurement. The energy of electrons is determined from a combination of the electron momentum at the primary interaction vertex is determined by the tracker, the energy of the corresponding ECAL cluster, and the energy sum of all bremsstrahlung photons spatially compatible with originating from the electron track. The energy of muons is obtained from the curvature of the corresponding track. The energy of charged hadrons is determined from a combination of their momentum measured in the tracker and the matching ECAL and HCAL energy deposits, corrected for zero-suppression effects and for the response function of the calorimeters to hadronic showers. Finally, the energy of neutral hadrons is obtained from the corresponding corrected ECAL and HCAL energies.

In the analysis, three main categories are defined: $0 \mathrm{~L}, 1 \mathrm{~L}$, and $2 \mathrm{~L}$ according to the reconstruction of 0,1 and 2 leptons (e or $\mu$ ) in the final state respectively. Furthermore, the $2 \mathrm{~L}$ category is subdivided into two subcategories via selections on the vector boson $\mathrm{p}_{\mathrm{T}}$ : $2 \mathrm{~L}-\mathrm{Low}-\mathrm{p}_{\mathrm{T}}(\mathrm{V})$ with a $\mathrm{p}_{\mathrm{T}}(\mathrm{V})$ in $[50,150] \mathrm{GeV}$ and $2 \mathrm{~L}-\mathrm{High}-\mathrm{p}_{\mathrm{T}}(\mathrm{V})$ with a $\mathrm{p}_{\mathrm{T}}(\mathrm{V})>150 \mathrm{GeV}$. The Higgs boson candidate is reconstructed considering the four-vectors of the leading and subleading b-tagged jets in the event. To tag efficiently the jets originating from the hadronization of the bottom quarks, a tagger based on a Deep Neural Network (DNN) architecture (DeepCSV) [15] has been deployed. This multiclassification algorithm takes different input features like the tracks and secondary vertex information, as well as the kinematics of the jets reconstructed through the particle-flow algorithm [14], and returns the probability that a jet is originating from a bottom, charm, or light 
quarks and gluons. One of the main improvements brought to the analysis performed with the data collected during 2017 is the improvement of the Higgs boson candidate invariant mass resolution. This has been achieved through two techniques: a dedicated bottom quark energy regression and a kinematic fit in the $2 \mathrm{~L}$ category. Because of the electroweak decays of the b-mesons originating from the b-quark hadronization, b-jets see the presence of neutrinos inside the jet cone and this leads to an underestimation of the jet energy and, ultimately, of the di-jet system invariant mass. A DNN-based algorithm has been developed by CMS to correct the energy of a jet for the energy carried out by the undetectable neutrinos [16]. The application of the b-jet energy regression within the $\mathrm{VH}(\mathrm{H} \rightarrow \mathrm{b} \bar{b})$ analysis categories led to an amelioration of the di-jet invariant mass resolution of $\sim 20 \%$. Furthermore, in events belonging to the $2 \mathrm{~L}$ category, it is possible to fully reconstruct the four-momentum of the vector boson relying on precise measurements of leptons observable. Constraining the di-lepton system to the $\mathrm{Z}$ boson mass and allowing the missing transverse energy in the events to float within the experimental uncertainties, the $\mathrm{p}_{\mathrm{T}}$ balance in the transverse plane of the vector sum of the two b-jets, the two leptons, and possible reconstructed additional jets is constrained to be null. This technique improves the measurement of the jet $\mathrm{p}_{\mathrm{T}}$, leading to an amelioration of the di-jet invariant mass resolution for events in the $2 \mathrm{~L}$ category of up to $\sim 35 \%$.

Another crucial aspect of the $\mathrm{CMS} \mathrm{VH}(\mathrm{H} \rightarrow \mathrm{b} \bar{b})$ search relies on the background estimation. It is indeed very difficult to predict precisely the different flavor composition of the jets originated in the $\mathrm{V}+\mathrm{jets}$ processes and contaminating the $\mathrm{H} \rightarrow \mathrm{b} \overline{\mathrm{b}}$ signal in simulation. Indeed the dominant background is coming mainly from the $\mathrm{V}+2 \mathrm{~b}$ processes and from the diboson production, where at least two jets originating from bottom quarks are present in the final state. While the diboson normalization is taken from simulation (the $\mathrm{Z}$ and $\mathrm{W}$ boson decays into b-quarks are known), to estimate the normalization of the various $\mathrm{V}+\mathrm{jets}$ processes a fit to data is required. Different control regions are defined in the analysis to provide enrichment in a particular $\mathrm{V}+\mathrm{jet}$ process according to the number of $b$-jet reconstructed in the final state. Finally, the signal extraction is performed through a binned maximum likelihood fit to all the signal and control regions simultaneously. To further increase the final signal over background discrimination, DNNs are used both in the signal region and in some of the control regions to differentiate the various $\mathrm{V}+\mathrm{jets}$ contributions from $\mathrm{tt}+\mathrm{jets}$ processes and the $\mathrm{VH}(\mathrm{H} \rightarrow \mathrm{b} \overline{\mathrm{b}})$ signal.

\subsection{ATLAS search for $\mathrm{VH}(\mathrm{H} \rightarrow \mathrm{b} \bar{b})$}

The analysis performed by ATLAS [9] to search for the $\mathrm{VH}(\mathrm{H} \rightarrow \mathrm{b} \bar{b})$ process with the data collected during 2017, relies, as the CMS analysis, on an extensive categorization of the events according to the reconstructed charged lepton multiplicity. There are three categories defined: $0 \mathrm{~L}$, $1 \mathrm{~L}$, and $2 \mathrm{~L}$ when the number of reconstructed electrons or muons is, in order, 0,1 , and 2 , aiming to target the $\mathrm{ZH} \rightarrow v v \mathrm{~b} \overline{\mathrm{b}}, \mathrm{WH} \rightarrow v \ell \mathrm{b} \overline{\mathrm{b}}$ and $\mathrm{ZH} \rightarrow \ell \ell \mathrm{b} \overline{\mathrm{b}}$ processes, respectively. In all channels, events are required to have exactly two b-tagged jets, which form the Higgs boson candidate. At least one b-tagged jet is required to have $\mathrm{p}_{\mathrm{T}}>45 \mathrm{GeV}$. Events are further split into 2-jet or 3-jet categories depending on whether additional, untagged jets are present. In the $0 \mathrm{~L}$ and $1 \mathrm{~L}$ channels, only one such jet is allowed, as the $\mathrm{t} t+\mathrm{jets}$ background is much larger in events with four jets or more. In the 2-lepton channel, any number of jets is accepted in the 3-jet category. The reconstructed transverse momentum $\mathrm{p}_{\mathrm{T}}(\mathrm{V})$ of the vector boson corresponds to the missing transverse 
energy in the $0 \mathrm{~L}$ category, to the vector sum of the missing transverse energy and the chargedlepton transverse momentum in the $1 \mathrm{~L}$ category, and to the transverse momentum of the 2-lepton system in the $2 \mathrm{~L}$ category. Similarly to the category definitions carried out in the CMS analysis, also in the ATLAS search the $2 \mathrm{~L}$ category is further subdivided into two subcategories according to the $\mathrm{p}_{\mathrm{T}}(\mathrm{V})$ value: $75<\mathrm{p}_{\mathrm{T}}(\mathrm{V})<150 \mathrm{GeV}$ and $\mathrm{p}_{\mathrm{T}}>150 \mathrm{GeV}$.

The Higgs boson candidate is reconstructed considering the four-vectors of the leading and subleading b-tagged jets in the event. To tag efficiently the jets originating from the hadronization of the bottom quarks, a tagger based on a Boosted Decision Tree (BDT) architecture (MV2) [17] has been deployed. In addition to the standard jet energy scale calibration [18], jets tagged as initiated by b-hadrons receive additional flavor-specific corrections to improve the measurement of their energy scale and resolution. In particular, corrections that account for the energy carried by soft-muons produced in the decays of the B-hadrons are added to the jet energy. In the 2L category, a per-event kinematic likelihood is deployed to improve the estimate of the b-tagged jets relying on the full event kinematic reconstruction. The amelioration of the di-jet invariant mass resolution achieves $\sim 40 \%$. Multivariate discriminants, based on BDT algorithms, are trained with variables that describe the kinematics of the selected events and used to maximize the sensitivity to the Higgs boson signal. Their output distributions are combined using a binned maximum-likelihood fit, referred to as the global likelihood fit, which allows the signal yield and the background normalizations to be extracted. There are 6 control regions defined to constrain the normalization of the $t \mathrm{t}+\mathrm{jets}$ and $\mathrm{V}+\mathrm{jets}$ processes, where, in the latter, the jets originate from heavy-flavor quarks.

\subsection{Results}

The CMS and ATLAS results obtained with the Run-1 and Run 2 data are summarized in Tab. 1. In both the analyses, the precision of the measurement of the signal strength $(\sim 20 \%)$ is limited by the systematic uncertainties. Among them, the most important are found to be those related to the b-tagging efficiencies, the background modeling, and the background normalization.

Table 1: Summary of the ATLAS [9] and CMS [10] results of the search for the $\mathrm{H} \rightarrow \mathrm{b} \bar{b}$ decay.

\begin{tabular}{l|ccc|ccc}
\hline \hline \multirow{2}{*}{ Data set } & \multicolumn{3}{|c|}{ ATLAS } & \multicolumn{3}{c}{ CMS } \\
& $\sigma($ Exp. $)$ & $\sigma($ Obs. $)$ & Signal strength $(\mu)$ & $\sigma($ Exp. $)$ & $\sigma($ Obs. $)$ & Signal strength $(\mu)$ \\
\hline $\mathrm{VH}(\mathrm{H} \rightarrow \mathrm{b} \overline{\mathrm{b}})$ Run 2 & 4.3 & 4.9 & $1.16 \pm 0.27$ & 4.2 & 4.4 & $1.06 \pm 0.26$ \\
$\mathrm{VH}(\mathrm{H} \rightarrow \mathrm{b} \overline{\mathrm{b}})$ Run-1+2 & 5.1 & 4.9 & $0.98 \pm 0.22$ & 4.9 & 4.8 & $1.01 \pm 0.23$ \\
$\mathrm{H} \rightarrow \mathrm{b} \overline{\mathrm{b}}$ Run-1+2 & 5.5 & 5.4 & $1.01 \pm 0.20$ & 5.5 & 5.6 & $1.04 \pm 0.20$ \\
\hline \hline
\end{tabular}

The ATLAS collaboration, besides, has performed also a combination of the $\mathrm{VH}(\mathrm{H} \rightarrow \mathrm{b} \overline{\mathrm{b}}) \mathrm{Run}$ 2 results with those from other Run 2 searches for the Higgs boson produced in the VH production mode, but decaying into either two photons or four leptons via $\mathrm{ZZ}^{*}$ decays. This combination led to the observation of the $\mathrm{VH}$ production mode with an observed (expected) significance of 5.3 (4.8) standard deviations $(\sigma)$. The ATLAS collaboration also provided the first reinterpretation of the $\mathrm{VH}(\mathrm{H} \rightarrow \mathrm{b} \bar{b})$ results in the context of the Simplified Template Cross-Section framework [19], measuring the production cross-section in bins of $\mathrm{p}_{\mathrm{T}}(\mathrm{V})$ separately for the $\mathrm{WH}$ and $\mathrm{ZH}$ modes. Indeed, modification of the cross-section in bins of $\mathrm{p}_{\mathrm{T}}(\mathrm{V})$ could highlight the contribution from new physics phenomena. The results are shown in Fig. 3. All bins have an observed (expected) 
significance between one and two standard deviations. With the amount of data available to date, these results are still dominated by the statistical uncertainty.

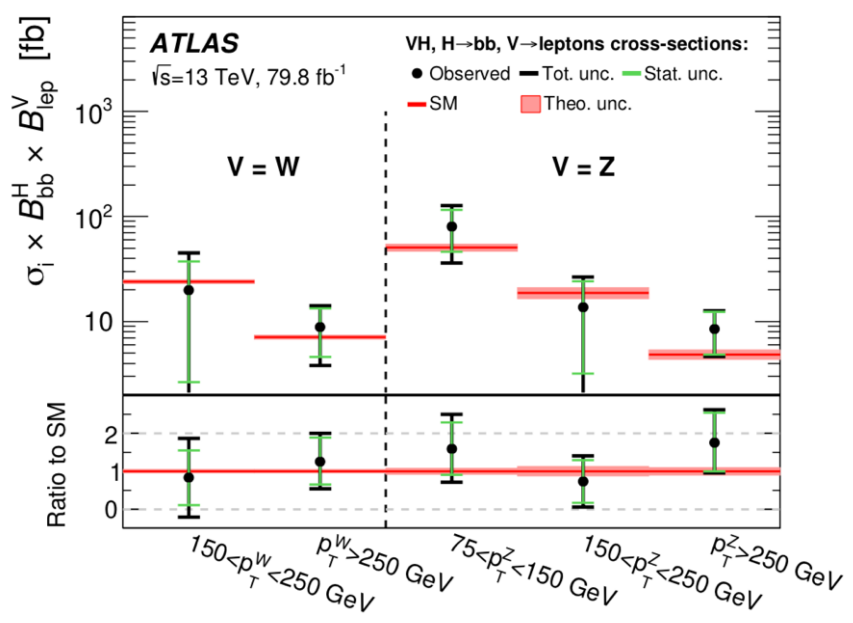

Figure 3: Measured $\mathrm{VH}, \mathrm{V} \rightarrow$ leptons reduced stage- 1 simplified template cross sections times the $\mathrm{H} \rightarrow \mathrm{b} \overline{\mathrm{b}}$ branching ratio [19].

\section{Higgs boson decay to $\mathrm{W}$ bosons}

The Higgs boson decay to a pair of W bosons was studied by the ATLAS and CMS Collaborations using the 7 and $8 \mathrm{TeV}$ data sets in leptonic final states, exploring several production mechanisms $[20,21,22]$. The probability of observing a signal at least as large as the one measured, under the background-only hypothesis, corresponded to an observed (expected) significance of 6.1 (5.8) $\sigma$ for ATLAS and CMS respectively. A later CMS combination [23], which includes Higgs boson production in association with a top-quark pair, reported an observed significance of $4.7 \sigma$ for this decay. Gluon fusion $(\mathrm{ggH})$ is the dominant production mode for a Higgs boson with a mass of $125 \mathrm{GeV}$ at $\sqrt{s}=13 \mathrm{TeV}$. The large Higgs boson branching fraction to a $\mathrm{W}$ boson pair makes this channel suitable for precision measurement of the Higgs boson production cross-section, and also allows studies of subleading production channels, such as Higgs boson production via vector boson fusion (VBF) and associated production with a vector boson $(\mathrm{VH})$.

\subsection{CMS search for $\mathrm{VH}(\mathrm{H} \rightarrow \mathrm{WW})$}

The search carried out by the CMS Collaboration [24] targeting Higgs bosons produced in association with a vector boson and decaying in a $\mathrm{W}$ boson pair is performed categorizing the events in three different classes according to the number and/or flavor of the reconstructed jets and charged leptons in the final state. The three- and four-lepton categories aim to reconstruct events where the Higgs boson decaying into $\mathrm{WW}$ is produced in association with a $\mathrm{W}$ or a $\mathrm{Z}$ respectively, while the 2-jet VH-tagged category targets final states where one vector boson ( $\mathrm{W}$ or $\mathrm{Z}$ ) decays into two resolved jets. This category with hadronically decaying vector bosons is affected by large backgrounds compared to the leptonic decays, but profits from a higher branching fraction. The 
2-jet VH-tagged analysis requires the di-jet invariant mass $\left(\mathrm{m}_{\mathrm{jj}}\right)$ to be between 65 and $105 \mathrm{GeV}$. Also, the two leading jets are required to have $|\eta|<2.5$ to profit from more stringent b jet veto requirements ( $b$ tagging can only be performed for central jets). To suppress $t++j e t s$ background, a selection on the two-lepton separation in the $\eta-\phi$ plane is applied, taking advantage of the spin- 0 nature of the Higgs boson that results in leptons being preferentially emitted in nearby directions.

The three-lepton WH-tagged analysis selects events that have the leading, subleading and training lepton $\mathrm{p}_{\mathrm{T}}$ above 25,20 and $15 \mathrm{GeV}$ respectively. Events with a fourth lepton with $\mathrm{p}_{\mathrm{T}}>10 \mathrm{GeV}$ are discarded. A veto is applied to events with same flavour lepton pairs of opposite charge that are compatible with coming from the $\mathrm{Z}$ boson decay. Events containing jets with $\mathrm{p}_{\mathrm{T}}>30 \mathrm{GeV}$ or b-tagged jets with $\mathrm{p}_{\mathrm{T}}>20 \mathrm{GeV}$ are also vetoed, to suppress the $t+\mathrm{t}$ ets background. The azimuthal angle between the missing transverse energy and the transverse momentum of the three-lepton system is used to reduce the contamination of non-prompt lepton backgrounds. In this category and the 2-jet VH-tagged one, the signal yield is extracted from the shape of the di-lepton mass distribution.

The ZH final state is targeted by requiring exactly four isolated leptons with tight identification criteria and zero total charge, and large missing transverse energy from the undetected neutrinos. The major background processes are $\mathrm{ZZ}$ and $\mathrm{ttZ}$ production. Among the four leptons, the pair of same flavor leptons with an opposite charge, and with the invariant mass closest to the $\mathrm{Z}$ boson one, is chosen as the $\mathrm{Z}$ boson candidate. Given the low expected signal yields, the result in this category is extracted from a simple event-counting.

\subsection{ATLAS search for $V H(H \rightarrow W \bar{W})$}

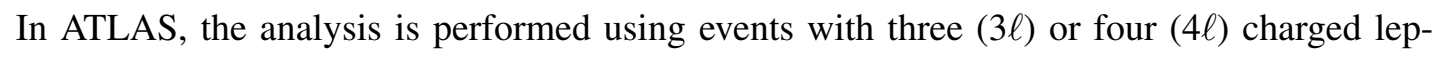
tons (electrons or muons) in the final state, targeting the $\mathrm{WH}$ and $\mathrm{ZH}$ channels respectively [25]. Leptonic decays of $\tau$ leptons from $\mathrm{H} \rightarrow \mathrm{WW}^{*} \rightarrow \tau \nu \tau \nu$ or $\mathrm{H} \rightarrow \mathrm{WW}^{*} \rightarrow \tau \nu \ell \nu$ decays are considered as signal, while no specific selection is performed for events with hadronically decaying $\tau$ leptons in the final state. Events from $\mathrm{VH}$ production with $\mathrm{H} \rightarrow \tau \tau$ are considered as background.

In the WH channel, exactly three isolated leptons with $\mathrm{p}_{\mathrm{T}}>15 \mathrm{GeV}$ are required with a total charge of \pm 1 . The most prominent background processes to the $\mathrm{WH}$ channel are $\mathrm{WZ} / \mathrm{W} \gamma^{*}$ production and top-quark processes. Other important background processes are $\mathrm{ZZ}^{*}$ (including $\left.\mathrm{Z} \gamma^{*}\right), \mathrm{Z} \gamma$ and $\mathrm{Z}+\mathrm{jets}$ production. The background from top-quark production is suppressed by vetoing events if they contain any b-tagged jet. The analysis of the WH channel separates events with at least one same-flavor opposite-sign charged lepton pair from events with zero lepton pairs, which have different signal-to-background ratios. A discriminant based on a BDT [25] is used to achieve a further separation between signal and background processes. The main purpose of the multivariate classifier is to distinguish between the signal and the dominant background processes. The BDT uses seven input variables. They are the magnitude of the vector sum of lepton transverse momenta, the invariant masses of the first lepton pair $\left(\mathrm{m}_{\ell \ell}\right)$ and of the three leptons $\left(\mathrm{m}_{\ell \ell \ell}\right)$, the angular distance between the lepton with unique charge and lepton closest to it, missing 
transverse energy, the pseudorapidity separation between the leptons with the same charge, and the transverse mass of the $\mathrm{W}$ boson. The signal region, defined as the events with high-ranking BDT score (BDT $>0.3$ ), is divided into three bins with increasing sensitivity: $0.3 \leq \mathrm{BDT}<0.5$, $0.5 \leq \mathrm{BDT}<0.7$ and $0.7 \leq \mathrm{BDT}<1.0$.

In the WH channel, multivariate discriminants are used to maximize the sensitivity to the Higgs boson signal, while in the $\mathrm{ZH}$ channel the analysis is performed through selection requirements. The distribution of these WH discriminants, together with event counts in background control regions and the signal regions in the $\mathrm{ZH}$ channel, are combined in a binned maximum-likelihood fit to extract the signal yield and the background normalizations. The maximum-likelihood fit provides results for the $\mathrm{WH}$ and the $\mathrm{ZH}$ channels separately and for their combination $\mathrm{VH}$, assuming the SM prediction for the relative cross-sections of the two production processes.

\subsection{Results}

The results in terms of signal strength $(\mu)$ of the ATLAS and the CMS searches are summarized in Tab. 2. Simultaneous fits are performed to probe the Higgs boson couplings to fermions and vector bosons and also to provide a comparison of the measured production cross-section for the $\mathrm{WH}$ and $\mathrm{ZH}$ processes. Both the experiments show that the results obtained are compatible with the SM expectation, as shown in Fig. 4.

Table 2: Summary of the ATLAS [25] and CMS [24] results of the search for the $\mathrm{H} \rightarrow \mathrm{WW}$ decay.

\begin{tabular}{l|c|c|}
\hline \hline \multirow{2}{*}{ Process } & \multicolumn{2}{|c|}{ Signal strength $(\mu)$} \\
& ATLAS & CMS $)$ \\
\hline $\mathrm{ZH}\left(\mathrm{H} \rightarrow \mathrm{W}^{+} \mathrm{W}^{-}\right)$ & $2.9_{-1.3}^{+1.9}$ & $1.00_{-1.00}^{+1.57}$ \\
$\mathrm{WH}\left(\mathrm{H} \rightarrow \mathrm{W}^{+} \mathrm{W}^{-}\right)$ & $2.3_{-1.0}^{+1.2}$ & $3.27_{-1.70}^{+1.88}$ \\
\hline \hline
\end{tabular}

\section{Higgs boson decay to tau lepton pair}

The Higgs boson decay to a $\tau$ lepton pair has the largest branching fraction among the leptonic Higgs boson decays $(\mathscr{B}(\mathrm{H} \rightarrow \tau \tau)=6.3 \%)$. The ATLAS and CMS Collaborations each previously reported evidence for this particular Higgs boson decay process using data collected at center-ofmass energies of 7 and $8 \mathrm{TeV}[26,27,28]$. The $\mathrm{H} \rightarrow \tau \tau$ process was measured targeting the gluon fusion and vector boson fusion production modes using data collected by the CMS Collaboration at a center-of-mass energy of $13 \mathrm{TeV}$ [29] resulting in a cross-section times branching fraction of $1.09_{-0.26}^{+0.27}$ relative to the SM expectation. The $\mathrm{H} \rightarrow \tau \tau$ decay is the second most sensitive channel to establish $\mathrm{VH}$ production, after the $\mathrm{VH}(\mathrm{H} \rightarrow \mathrm{b} \bar{b})$ process.

The $\tau$ lepton can decay to an electron, a muon, or hadrons. The hadronic decays of the $\tau$ lepton represent $\sim 64 \%$ of its total width. Typically $\tau_{\mathrm{h}}$ decays into either one or three charged mesons (predominantly $\pi^{+} \pi^{-}$) in the presence of up to two neutral pions, decaying via $\pi^{0} \rightarrow \gamma \gamma$. Hadronically decaying $\tau$ leptons $\left(\tau_{\mathrm{h}}\right)$ are reconstructed with the hadron-plus-strips (HPS) algorithm [30, 31], taking in input jets clustered with the anti-kt algorithm [32, 33] with a distance 

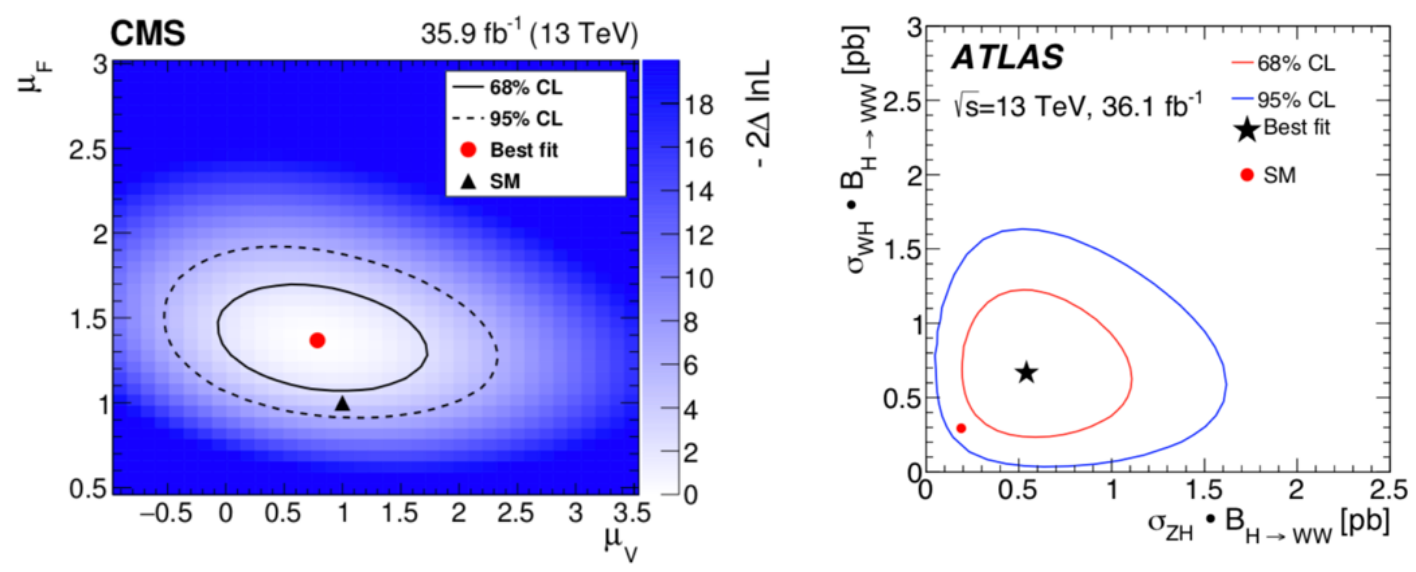

Figure 4: Left: Two-dimensional likelihood profile as a function of the signal strength modifiers associated with either fermion $\left(\mu_{\mathrm{F}}\right)$ or vector boson $\left(\mu_{\mathrm{V}}\right)$ couplings obtained by the CMS search. The $68 \%$ and $95 \% \mathrm{CL}$ contours are shown as continuous and dashed lines, respectively. The red circle represents the best fit value, while the black triangle corresponds to the SM prediction [24]. Right: Two-dimensional likelihood contours of $\sigma_{\mathrm{WH}} \times \mathscr{B}(\mathrm{H} \rightarrow \mathrm{WW})$ vs. $\sigma_{\mathrm{ZH}} \times \mathscr{B}(\mathrm{H} \rightarrow \mathrm{WW})$ for $68 \%$ and $95 \%$ confidence level (CL) compared with the prediction from the SM [25].

parameter of 0.4. The HPS algorithm reconstructs $\tau_{\mathrm{h}}$ candidates on the basis of the number of tracks and on the number of ECAL strips with an energy deposit in the $\eta-\phi$ plane, in the 1-prong, 1-prong $+\pi^{0}$, and 3-prong decay modes, where prong stands for a charged meson $\left(\pi^{ \pm}, \mathrm{k}^{ \pm}\right)$originating directly from the hadronic decays of the $\tau$ lepton. A discriminator based on a multivariate analysis technique, including information on the lifetime and isolation, is used to reduce the rate for quark- and gluon-initiated jets to be identified as $\tau_{\mathrm{h}}$ candidates.

In all final states, the visible mass of the Higgs boson candidate, $\mathrm{m}_{\mathrm{vis}}$, can be used to separate the $\mathrm{H} \rightarrow \tau \tau$ signal events from the large irreducible contribution of $\mathrm{Z} \rightarrow \tau \tau$ events. However, the neutrinos from the $\tau$ lepton decays carry a large fraction of the $\tau$ lepton energy and reduce the discriminating power of this variable. The SVFit algorithm [34] combines the missing transverse momentum vector with the four-vector momenta of both $\tau$ candidates to estimate the mass of the parent boson $\left(\mathrm{m}_{\tau \tau}\right)$. The resolution of $\mathrm{m}_{\tau \tau}$ is about $20 \%$. The $\mathrm{m}_{\tau \tau}$ variable is used for the $\mathrm{ZH}$ channels, while the invariant mass of the visible $\tau_{\mathrm{h}}$ is used in the $\mathrm{WH}$ channels due to the impossibility for the SVFIT algorithm to account for the additional missing energy taken from the neutrino coming from the $\mathrm{W}$ boson decay.

In order to target the $\mathrm{WH}$ production mode the decay channels analyzed are: $\mathrm{W}(\mathrm{e} v) \mathrm{H}\left(\mu \tau_{\mathrm{h}}\right)$, $\mathrm{W}(\mu v) \mathrm{H}\left(\mu \tau_{\mathrm{h}}\right), \mathrm{W}(\mathrm{e} v) \mathrm{H}\left(\tau_{\mathrm{h}} \tau_{\mathrm{h}}\right), \mathrm{W}(\mu v) \mathrm{H}\left(\tau_{\mathrm{h}} \tau_{\mathrm{h}}\right)$, while to target the $\mathrm{ZH}$ production mode the $\mathrm{Z}$ boson is reconstructed through its decay to two electrons or two muons and the Higgs boson decays to two $\tau$ leptons is reconstructed through the final states $\mathrm{H}(\mathrm{e} \mu), \mathrm{H}\left(\mathrm{e} \tau_{\mathrm{h}}\right), \mathrm{H}\left(\mu \tau_{\mathrm{h}}\right), \mathrm{H}\left(\tau_{\mathrm{h}} \tau_{\mathrm{h}}\right)$. Event categories are defined by three-lepton final states targeting WH production, and four-lepton final states targeting $\mathrm{ZH}$ production. The irreducible backgrounds are $\mathrm{WZ}$ or $\mathrm{ZZ}$ depending on the Higgs 
boson production process targeted and they are estimated from simulation. Instead, to evaluate the $\mathrm{tt}+\mathrm{jets}$ and $\mathrm{V}+\mathrm{jets}$ background a data-driven method has been deployed (fake-rate method [35]).
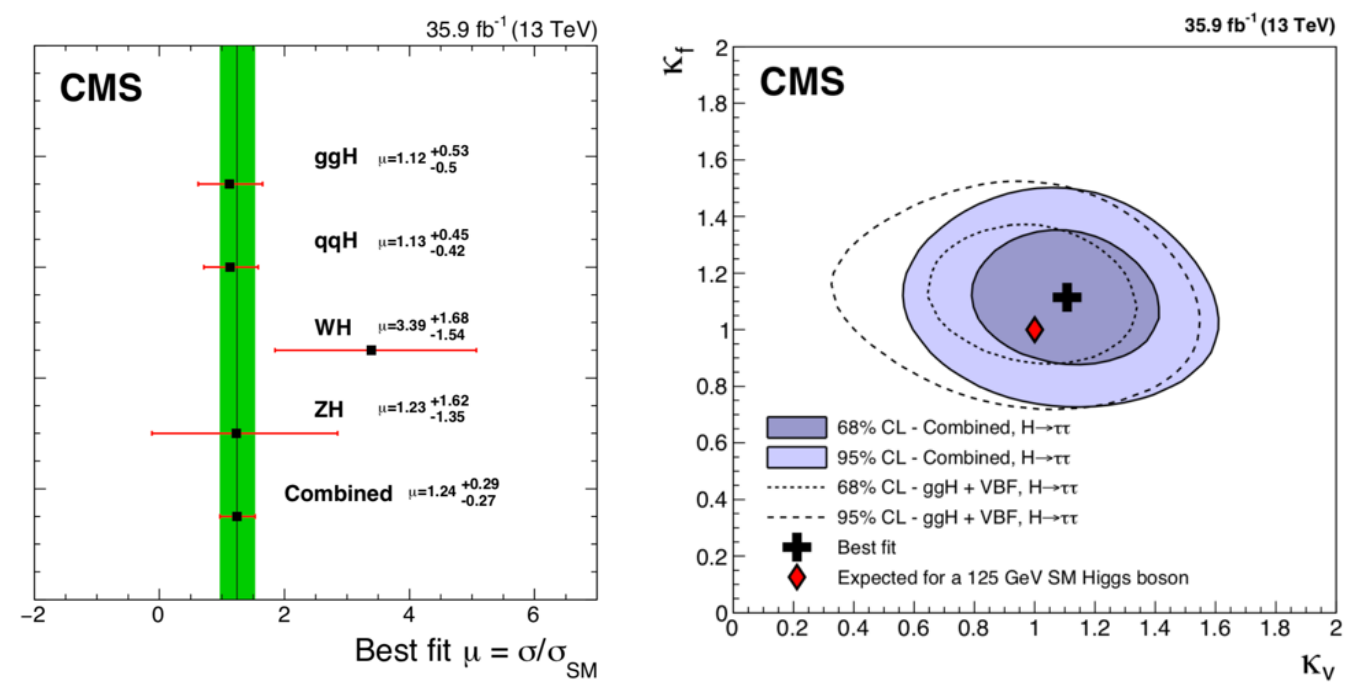

Figure 5: Left: Best fit signal strength per Higgs boson production process, for $m_{H}=125 \mathrm{GeV}$, using a combination of the $\mathrm{WH}$ and $\mathrm{ZH}$ targeted analysis with the CMS analysis performed in the same data set for the same decay mode but targeting the gluon fusion and vector boson fusion production mechanisms [35]. Right: Scans of the negative log-likelihood difference as a function of $\kappa_{V}$ and $\kappa_{f}$, for $\mathrm{m}_{\mathrm{H}}=125 \mathrm{GeV}$. Contours corresponding to confidence levels (CL) of 68 and 95\% are shown [35].

The signal strength resulting from the best maximum likelihood fit to the $\mathrm{WH}$ and $\mathrm{ZH}$ associated production event distributions is $\mu=2.5_{-1.3}^{+1.4}\left(1.0_{-1.0}^{+1.1}\right.$ expected) with a significance of 2.3 standard deviations (1.0 expected) [35]. The results of this analysis are combined with those of the CMS analyses targeting gluon fusion and vector boson fusion production, also performed at a center-of-mass energy of $13 \mathrm{TeV}$, and constraints on the $\mathrm{H} \rightarrow \tau \tau$ decay rate are set. The best fit signal strength is $\mu=1.24_{-0.27}^{+0.29}\left(1.00_{-0.23}^{+0.24}\right.$ expected $)$, and the observed significance is 5.5 standard deviations (4.8 expected) for a Higgs boson mass of $125 \mathrm{GeV}$, as reported in Fig. 5. This combination further constrains the coupling of the Higgs boson to vector bosons, resulting in measured couplings that are consistent with SM predictions within one standard deviation, as also shown in Fig. 5. The combination allows for the extraction of the signal strengths for the four leading Higgs boson production processes using exclusively $\mathrm{H} \rightarrow \tau \tau$ targeted final states, the results of which are largely consistent with the SM. The measurements of the Higgs boson production mechanisms using $\mathrm{H} \rightarrow \tau \tau$ decays are the best results to date for the $\mathrm{WH}$ and $\mathrm{ZH}$ associated production mechanisms exploiting the $\mathrm{H} \rightarrow \tau \tau$ decay mode.

\section{Higgs boson decay to charm quark pairs}

The ATLAS collaboration has published in 2016 the first direct search at the LHC for the Higgs boson decay into a pair of charm quarks [36]. The search is performed using pp collision data recorded in 2015 and 2016 with the ATLAS detector at $\sqrt{s}=13 \mathrm{TeV}$, corresponding to a total 
integrated luminosity of $36.1 \mathrm{fb}^{-1}$. The analysis targets events of Higgs bosons produced in association with a $\mathrm{Z}$ boson. The analysis strategy is based on the identification of the jets originating from the charm quarks thanks to the usage of a dedicated charm tagger capable of exploiting the different life-time of charm, bottom, and light-flavor quark or gluon jets. Two BDTs are trained in order to discriminate $\mathrm{c}$ jets from $\mathrm{b}$ jets and $\mathrm{c}$ jets from light-flavor quark or gluon jets.

The events are triggered exploiting the single-lepton trigger benefiting from the presence of two electrons and two muons from the leptonic decay of the $\mathrm{Z}$ boson. The four-momentum of the reconstructed electrons and muons are used to build the four-momentum of the $\mathrm{Z}$ boson candidate, while the Higgs boson candidate four-momentum is computed as the sum of the four-momenta of the two jets reconstructed from topological clusters in the calorimeters, using the anti-kt algorithm $[32,33]$ with distance parameter 0.4 . The energy of the jets is corrected using a jet-area-based technique and calibrated with $\mathrm{p}_{\mathrm{T}^{-}}$and $\eta$-dependent correction factors. Events with at least two jets with $\mathrm{p}_{\mathrm{T}}>20 \mathrm{GeV}$ and $|\eta|<2.4$ are selected. The invariant mass of the reconstructed leptons is required to be compatible within 81 and $101 \mathrm{GeV}$.
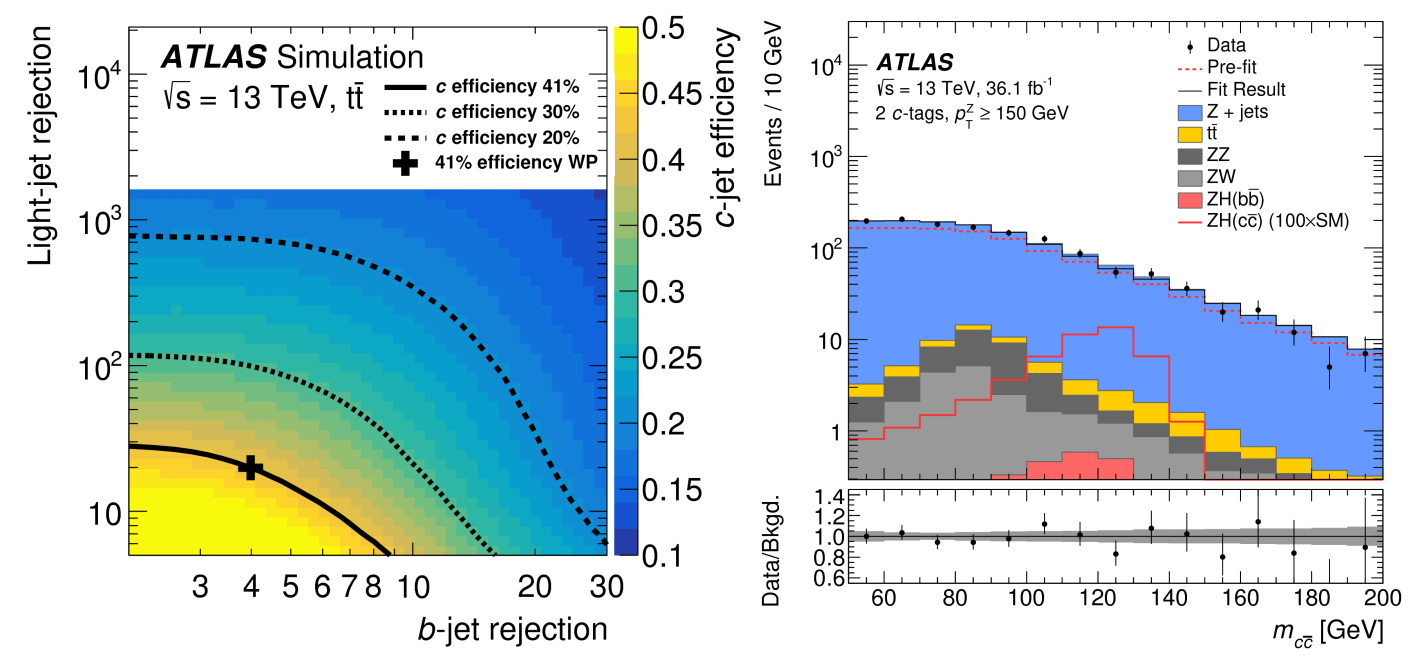

Figure 6: Left: The $\mathrm{c}$ jet tagging efficiency (colored scale) as a function of the $\mathrm{b}$ jet and light-flavor quark or gluon jet rejection as obtained from simulated $t \bar{t}+j e t s$ events. The black cross indicates the working point used in the ATLAS analysis and the lines are c-tag iso efficiency curves [36]. Right: Observed and predicted $\mathrm{m}_{\mathrm{c} \overline{\mathrm{c}}}$ distributions in the 2c-tag analysis categories [36].

The analysis strategy aims to categorize the events to increase the sensitivity to the $\mathrm{VH}(\mathrm{H} \rightarrow \mathrm{c} \overline{\mathrm{c}})$ process. Data are analyzed in four categories with different expected signal purity based on the $\mathrm{Z}$ boson candidate reconstructed $\mathrm{p}_{\mathrm{T}}\left(75<\mathrm{p}_{\mathrm{T}}(\mathrm{Z})<150 \mathrm{GeV}\right.$ and $\left.\mathrm{p}_{\mathrm{T}}(\mathrm{Z})>150 \mathrm{GeV}\right)$ and on the number of jets identified as originating from a charm quark (i.e. passing a cut on the two-dimensional charm tagger discriminator). The $\mathrm{c}$ jet tagging efficiency as a function of the $\mathrm{b}$ jet and lightflavor quark or gluon rejection rate is shown in Fig. 6. The working point employed in the ATLAS $\mathrm{VH}(\mathrm{H} \rightarrow \mathrm{c} \overline{\mathrm{c}})$ analysis allows for a $\mathrm{c}$ jet identification efficiency of approximatively $41 \%$ with a b jet and light-flavor quark or gluon rejection rate of 4 and 20 respectively. Finally, the signal extraction 
is performed through a binned maximum likelihood fit to the di-jet invariant mass $\left(m_{c \bar{c}}\right)$ in all the analysis categories simultaneously to extract the signal yield and the $Z+j$ ets background normalization. An example of the fitted $m_{c \bar{c}}$ distribution is provided in Fig. 6. The result in terms of observed (expected) $95 \%$ confidence level exclusion limits on the $\sigma(\mathrm{VH}) \times \mathscr{B}(\mathrm{H} \rightarrow \mathrm{c} \overline{\mathrm{c}})$ is $2.7\left(3.9_{-1.1}^{+2.1}\right) \mathrm{pb}$.

At the time of writing these proceedings, the above-mentioned measurement has been recently superseded by a new analysis carried out by the CMS collaboration [37]. A more aggressive analysis strategy has been adopted, exploiting multivariate analysis techniques and an enhanced categorization of the events. The result of the CMS analysis increases the expected significance by a factor four, providing the most stringent exclusion limit to date on the $\sigma(\mathrm{VH}) \times \mathscr{B}(\mathrm{H} \rightarrow \mathrm{c} \overline{\mathrm{c}})$, measured to be $4.5\left(2.4_{-0.7}^{+1.0}\right) \mathrm{pb}[37]$ at $95 \%$ confidence level.

\section{Conclusion}

In these proceedings, a review of the main searches targeting Higgs bosons produced through the associated production mechanism, as they are carried out by the ATLAS and CMS experiments at the LHC, is provided. Despite the lower cross-section of this Higgs boson production mode if compared to the gluon-fusion and vector-boson-fusion processes, it represents a powerful handle to be exploited to characterize the Higgs boson properties. In particular, it plays a crucial role in the searches for Higgs boson decays into third- and second-generation quarks, providing a unique experimental signature that allows a high fraction of the overwhelming multijet background arising from QCD processes to be rejected. In both the ATLAS and CMS observation of the Higgs boson decay into a bottom-antibottom quark pair, the analyses targeting the $\mathrm{VH}$ production mechanism drive the total sensitivity. The observation of this Higgs boson decay mode has been possible thanks to the exploitation of the events originating from the $\mathrm{VH}$ mechanism.

\section{References}

[1] Englert, F. and Brout, R., "Observation of a new particle in the search for the Standard Model Higgs boson with the ATLAS detector at the LHC", Phys. Rev. Lett. 13 (1964) 321-323 doi:10.1103/PhysRevLett.13.321

[2] Higgs, Peter W., "Broken Symmetries and the Masses of Gauge Bosons", Phys. Rev. Lett. 13 (1964) 508-509 doi:10.1103/PhysRevLett.13.508

[3] Guralnik, G. S. and Hagen, C. R. and Kibble, T. W. B., "Global Conservation Laws and Massless Particles”, Phys. Rev. Lett. 13 (1964) 585-587 doi:10.1103/PhysRevLett.13.585

[4] G. Aad et al. [ATLAS Collaboration], "Observation of a new particle in the search for the Standard Model Higgs boson with the ATLAS detector at the LHC," Phys. Lett. B 716 (2012) 1 doi:10.1016/j.physletb.2012.08.020

[5] S. Chatrchyan et al. [CMS Collaboration], "Observation of a New Boson at a Mass of $125 \mathrm{GeV}$ with the CMS Experiment at the LHC,” Phys. Lett. B 716 (2012) 30 doi:10.1016/j.physletb.2012.08.021

[6] Chatrchyan, S., et al. [CMS Collaboration], "Observation of a new boson with mass near $125 \mathrm{GeV}$ in pp collisions at $\sqrt{s}=7$ and 8TeV”, J. High Energ. Phys. 81 (2013) 2013:81 doi:https://doi.org/10.1007/JHEP06(2013)081 
[7] M. Aaboud et al. [ATLAS Collaboration], "Observation of Higgs boson production in association with a top quark pair at the LHC with the ATLAS detector", Phys. Lett. B 784 (2018) 173 doi:10.1016/j.physletb.2018.07.035

[8] Chatrchyan, S., et al. [CMS Collaboration], “Observation of $t \bar{t} H$ Production” Phys. Rev. Lett. 120 (2018) 231801 doi:10.1103/PhysRevLett.120.231801

[9] M. Aaboud et al. [ATLAS Collaboration], "Observation of $H \rightarrow b \bar{b}$ decays and $V H$ production with the ATLAS detector", Phys. Lett. B 786 (2018) 59 doi:10.1016/j.physletb.2018.09.013

[10] A. M. Sirunyan et al. [CMS Collaboration], "Observation of Higgs Boson Decay to Bottom Quarks, Phys. Rev. Lett. 121, 121801 (2018) doi:10.1103/PhysRevLett.121.121801

[11] G Aad et al. [ATLAS Collaboration], "The ATLAS Experiment at the CERN Large Hadron Collider", JINST 3 (2008) 08 S08003 doi:10.1088/1748-0221/3/08/s08003

[12] S Chatrchyan et al. [CMS Collaboration], "The CMS experiment at the CERN LHC", JINST 3 (2008) 08 S08004 doi:10.1088/1748-0221/3/08/s08004

[13] D. de Florian et al. [LHC Higgs Cross Section Working Group], "Handbook of LHC Higgs Cross Sections: 4. Deciphering the Nature of the Higgs Sector", doi:10.2172/1345634, 10.23731/CYRM-2017-002

[14] A. M. Sirunyan et al. [CMS Collaboration], "Particle-flow reconstruction and global event description with the CMS detector," JINST 12 (2017) no.10, P10003 doi:10.1088/1748-0221/12/10/P10003

[15] A. M. Sirunyan et al. [CMS Collaboration], "Identification of heavy-flavour jets with the CMS detector in pp collisions at 13 TeV", JINST 13 (2018) no.05, P05011, doi:10.1088/1748-0221/13/05/P05011

[16] CMS Collaboration [CMS Collaboration], "A deep neural network for simultaneous estimation of b quark energy and resolution", CMS-PAS-HIG-18-027. https : //cds.cern.ch/record/2690804

[17] M. Aaboud et al. [ATLAS Collaboration], "Optimisation and performance studies of the ATLAS $b$-tagging algorithms for the 2017-18 LHC run", ATL-PHYS-PUB-2017-013 http : //cds.cern.ch/record/2273281

[18] M. Aaboud et al. [ATLAS Collaboration], "Jet energy scale measurements and their systematic uncertainties in proton-proton collisions at $\sqrt{s}=13 \mathrm{TeV}$ with the ATLAS detector", Phys. Rev. D 96 (2017) no.7, 072002, doi:10.1103/PhysRevD.96.072002

[19] M. Aaboud et al. [ATLAS Collaboration], "Measurement of $V H, H \rightarrow b \bar{b}$ production as a function of the vector-boson transverse momentum in $13 \mathrm{TeV}$ pp collisions with the ATLAS detector", J. High Energ. Phys. 5 (2019) 2019:141, doi:https://doi.org/10.1007/JHEP05(2019)141

[20] G. Aad et al. [ATLAS Collaboration], "Observation and measurement of Higgs boson decays to WW* with the ATLAS detector", Phys. Rev. D 92 (2015) no.1, 012006 doi:10.1103/PhysRevD.92.012006

[21] G. Aad et al. [ATLAS Collaboration], "Study of (W/Z)H production and Higgs boson couplings using $H \rightarrow W W^{*}$ decays with the ATLAS detector", JHEP 1508 (2015) 137 doi:10.1007/JHEP08(2015)137

[22] S. Chatrchyan et al. [CMS Collaboration], "Measurement of Higgs boson production and properties in the WW decay channel with leptonic final states", JHEP 1401 (2014) 096 doi:10.1007/JHEP01(2014)096

[23] V. Khachatryan et al. [CMS Collaboration], "Precise determination of the mass of the Higgs boson and tests of compatibility of its couplings with the standard model predictions using proton collisions at 7 and 8 TeV", Eur. Phys. J. C 75 (2015) no.5, 212 doi:10.1140/epjc/s10052-015-3351-7 
[24] A. M. Sirunyan et al. [CMS Collaboration], "Measurements of properties of the Higgs boson decaying to a W boson pair in pp collisions at $\sqrt{s}=13 \mathrm{TeV}$ ", Phys. Lett. B 791 (2019) 96 doi:10.1016/j.physletb.2018.12.073

[25] G. Aad et al. [ATLAS Collaboration], "Measurement of the production cross section for a Higgs boson in association with a vector boson in the $H \rightarrow W W^{*} \rightarrow \ell v \ell v$ channel in pp collisions at $\sqrt{s}=13$ TeV with the ATLAS detector',' Phys. Lett. B 798 (2019) 134949 doi:10.1016/j.physletb.2019.134949

[26] G. Aad et al. [ATLAS Collaboration], "Evidence for the Higgs-boson Yukawa coupling to tau leptons with the ATLAS detector", JHEP 1504 (2015) 117 doi:10.1007/JHEP04(2015)117

[27] G. Aad et al. [ATLAS Collaboration], "Search for the Standard Model Higgs boson produced in association with a vector boson and decaying into a tau pair in $p p$ collisions at $\sqrt{s}=8 \mathrm{TeV}$ with the ATLAS detector", Phys. Rev. D 93 (2016) no.9, 092005 doi:10.1103/PhysRevD.93.092005

[28] S. Chatrchyan et al. [CMS Collaboration], "Evidence for the $125 \mathrm{GeV}$ Higgs boson decaying to a pair of $\tau$ leptons", JHEP 1405 (2014) 104 doi:10.1007/JHEP05(2014)104

[29] A. M. Sirunyan et al. [CMS Collaboration], "Observation of the Higgs boson decay to a pair of $\tau$ leptons with the CMS detector", Phys. Lett. B 779 (2018) 283 doi:10.1016/j.physletb.2018.02.004

[30] V. Khachatryan et al. [CMS Collaboration], "Reconstruction and identification of $\tau$ lepton decays to hadrons and $v_{\tau}$ at CMS", JINST 11 (2016) no.01, P01019 doi:10.1088/1748-0221/11/01/P01019

[31] V. Khachatryan et al. [CMS Collaboration], "Performance of reconstruction and identification of tau leptons in their decays to hadrons and tau neutrino in LHC Run 2", CMS-PAS-TAU-16-002 https : //cds.cern.ch/record/2196972

[32] M. Cacciari, G. P. Salam and G. Soyez, "The anti- $k_{t}$ jet clustering algorithm,” JHEP 0804 (2008) 063 doi:10.1088/1126-6708/2008/04/063

[33] M. Cacciari, G. P. Salam and G. Soyez, "FastJet User Manual," Eur. Phys. J. C 72 (2012) 1896 doi:10.1140/epjc/s10052-012-1896-2

[34] Lorenzo Bianchini and John Conway and Evan Klose Friis and Christian Veelken, "Reconstruction of the Higgs mass in $H \rightarrow \tau \bar{\tau}$ Events by Dynamical Likelihood techniques", Journal of Physics: Conference Series 2 (2014) 513, 022035 doi:10.1088/1742-6596/513/2/022035

[35] A. M. Sirunyan et al. [CMS Collaboration], "Search for the associated production of the Higgs boson and a vector boson in proton-proton collisions at $\sqrt{s}=13 \mathrm{TeV}$ via Higgs boson decays to $\tau$ leptons", JHEP 1906 (2019) 093 doi:10.1007/JHEP06(2019)093

[36] M. Aaboud et al. [ATLAS Collaboration], "Search for the Decay of the Higgs Boson to Charm Quarks with the ATLAS Experiment", Phys. Rev. Lett. 120 (2018) no.21, 211802 doi:10.1103/PhysRevLett.120.211802

[37] V. Khachatryan et al. [CMS Collaboration], "Search for the standard model Higgs boson decaying to charm quarks", CMS-PAS-HIG-18-031 (2019) https : //cds.cern.ch/record/2682638 